\title{
Influence of Freeze-Thaw Cycles on Tensile Strength and Fracture Toughness of Concrete
}

\author{
Lingtong MENG ${ }^{\mathrm{a}}$, Shutong $\mathrm{YANG}^{\mathrm{a}}$ and Song $\mathrm{YANG}^{\mathrm{a}, 1}$ \\ ${ }^{a}$ Department of Civil Engineering, College of Engineering, Ocean University of China, \\ Qingdao 266100, China
}

\begin{abstract}
In this paper, the fracture behavior of concrete with different initial notch lengths after freeze-thaw action was studied by using three-point bending test. Then, based on the boundary effect model, the parameters indicating the material discontinuity and inhomogeneity were introduced, and the maximum fracture load of the beam was used to determine the real tensile strength and fracture toughness of concrete under different freeze-thaw cycles. Results show that the tensile strength and fracture toughness of concrete are obviously reduced. Compared with the control specimens under indoor condition, the fracture parameters are reduced by more than $38 \%$ when the number of freeze-thaw cycles reached 75 times. In this paper, the tensile strength obtained based on the boundary effect model is significantly higher than the splitting tensile strength of concrete due to the incorporation of the discontinuity and non-uniformity of materials, and can more accurately reflect the deterioration and damage degree of concrete after freeze-thaw action.
\end{abstract}

Keywords. Three-point bending test; freeze-thaw cycle; fracture toughness; tensile strength; maximum fracture load

\section{Introduction}

In recent years, with the launch and implementation of China's "maritime power" strategy, China has vigorously promoted the construction of marine engineering. It is known in northern China that when temperature drops drastically, the durability of concrete structures in the freeze-thaw environment faces great challenges. Therefore, concrete buildings in the northern coastal areas are subjected to the erosion from freezing and thawing cycles round the year, which greatly reduce the durability of concrete. During the freeze-thaw cycle, internal damage developed in concrete. Hence, fracture mechanics based analysis of concrete becomes essential.

A lot of research work has been carried out on the variation in fracture properties of concrete under the freeze-thaw cycle. As for the deterioration law of concrete under the actions of carbonization, dry-wetting and freeze-thaw, $\mathrm{Gu}$ [1] found that the freezethaw cycle had the largest influence on the fracture performance of specimens. $\mathrm{Hu}$ [2] studied the influence of salt freeze-thaw, water freeze-thaw and some other freeze-thaw

${ }^{1}$ Song Yang, Department of Civil Engineering,College of Engineering, Ocean University of China, Qingdao 266100, China; E-mail: yangsong@ouc.edu.cn. 
environments on the double- $K$ fracture toughness of concrete, and found that the overall fracture performance of concrete was very sensitive to the salt freeze-thaw environment due to the simultaneous decrease of concrete's ability to resist crack formation and expansion in the salt freeze-thaw environment. Wang [3] conducted freeze-thaw cycle tests on concrete with different crack lengths, and found that the fracture toughness of concrete was independent of the initial crack lengths under the same number of cycles. Zhao et al. [4] carried out tests on freeze-thaw cycles and fracture properties of concrete mixed with basalt fibers, and found that the fracture toughness of concrete after the freeze-thaw cycles increased first and then decreased with increasing fiber content. However, the basalt fibers could not completely inhibit the damage caused by the freeze-thaw cycles.

By determining fracture parameters under different freeze-thaw cycles, the deterioration of concrete fracture performance can be analyzed quantitatively. Although some studies have been performed on the variation of fracture parameters under different freeze-thaw cycles, the parameters are mostly the double- $K$ fracture parameters $[5,6]$, which are determined based on linear elastic fracture mechanics. The traditional splitting tensile strength derived based onthe continuity and homogeneity is generally used to represent the tensile strength. But it is essentially the average theoretical tensile strength rather than the real tensile strength $f_{\mathrm{t}}$. Since concrete is a composite material composed of cementitious material and coarse and fine aggregate, it has obvious discontinuity and inhomogeneity in essence. Therefore, the fracture parameters obtained by traditional methods have significant size effect [7, 8]. Moreover, both internal damage and inhomogeneity of concrete increase with increasing freezethaw cycle. The continuity and inhomogeneity based on theory of linear elasticity is applicable only to concrete specimens having sufficient large size enough. But it is too difficult to be realized in ordinary laboratory. To better analyze the variation of mechanical properties of concrete with the number of freeze-thaw cycles, it is very important to find an appropriate method to determine the real mechanical parameters of concrete. In recent years, $\mathrm{Hu}$ et al. $[9,10]$ have developed the boundary effect model (BEM). Incorporating the average particle size for reflecting the material inhomogeneity [11], the real tensile strength $f_{\mathrm{t}}$ and fracture toughness $K_{\mathrm{IC}}$ without size effect can be obtained by using small and medium-sized size specimens under ordinary laboratory conditions, and only the maximum fracture load $F_{\max }$ of specimens is measured. Small concrete beams with initial notches were prepared and subjected to 25 , 50 and 75 freeze-thaw cycles. Three-point-bending tests were carried out for beams. Furthermore, based on the BEM [12], $f_{\mathrm{t}}$ and $K_{\mathrm{IC}}$ of concrete under different conditions were determined for evaluating the influence of the freeze-thaw cycles.

\section{Experimental Program}

\subsection{Materials and Mix Proportion}

Coarse aggregate is produced by Tianjin Yandong Mineral Products Co. Ltd, with sizes from $5 \mathrm{~mm}$ to $10 \mathrm{~mm}$.

Fine aggregate is river sand provided by the Qingdao flat production.

The water reducing agent is polycarboxylic acid produced by Qingdao Huatie New Materials Co. Ltd. The water reducing rate was $27 \%-29 \%$. 
Cement used in the test is P.O.42.5 ordinary Portland cement, produced by Shandong Shanshui Cement (Qingdao) Co. Ltd.

The mix proportion of the concrete mixture is shown in table 1 .

Table 1. Mix proportion of C55.

\begin{tabular}{llllllll}
\hline Water & Cement & A & F & $\begin{array}{l}\text { Coarse } \\
\text { aggregate }\end{array}$ & Fineaggregate & Waterreducer & Airentrainingagent \\
\hline 162 & 448 & 4 & 4 & 1058 & 470 & 3.68 & 0.0044 \\
\hline
\end{tabular}

\subsection{Test of Cubic Compressive Strength and Splitting Tensile Strength}

As per GB/T50081-2019, the test was carried out on cubic specimens of size $150 \mathrm{~mm} \times 150 \mathrm{~mm} \times 150 \mathrm{~mm}$. After curing for 28 days under standard conditions (temperature $20^{\circ}$ Cand relative humidity $95 \%$ ), the compressive strength $f_{\text {cu }}$ and splitting tensile strength $f_{\mathrm{ts}}$ of the specimens were determined. Three specimens were prepared in each group.

\subsection{Test of Freeze-thaw Cycle}

The test was based on the standard rapid freeze-thaw cycle as in GB/T50082-2009. It was carried out on a KDR-V9 rapid freeze-thaw test machine. Each cycle time was 4.5h. The temperature was controlled between minus 18 and plus 5 degrees ($\left.18 \pm 2^{\circ} \mathrm{C} \sim 5 \pm 2^{\circ} \mathrm{C}\right)$. The size of the small three-point-bending beams was $100 \mathrm{~mm} \times 100 \mathrm{~mm} \times 400 \mathrm{~mm}$. According to the number of freeze-thaw cycles, the specimens were divided into control groups (COM) as follows: freeze-thaw cycle 25 times (FT25), freeze-thaw cycle 50 times (FT50), and freeze-thaw cycle 75 times (FT75). Three $a_{0} / h$ ratios were considered, i.e., $0.1,0.2$ and 0.3 . Seven samples were tested for each $a_{0} / h$.

\subsection{Test of Three-point-bending Beam}

The three-point bending test was carried out on a YAW-100D electronic universal testing machine. The applied load was measured by a load cell with a maximum range $70 \mathrm{kN}$. The crack mouth opening displacement $(C M O D)$ was measured by a clip gauge with a maximum range of $4 \mathrm{~mm}$. The data were collected by a data acquisition system. The loading rate was set as $0.2 \mathrm{~mm} / \mathrm{min}$.

\section{Results and Discussions}

\subsection{Results of $f_{c u}$ and $f_{\text {ts }}$}

Table 2 shows the cubic compressive strength and splitting tensile strength of the concrete used in the test after curing for $28 \mathrm{~d}$ under standard curing conditions. Table 2 shows the mean values of each group of three samples, with standard deviations in parentheses. 
Table 2. Results of $f_{\text {cu }}$ and $f_{\text {ts. }}$.

\begin{tabular}{ll}
\hline$f_{\text {cu }}(\mathrm{MPa})$ & $f_{\text {ts }}(\mathrm{MPa})$ \\
\hline $58.8(0.25)$ & $5.03(0.08)$ \\
\hline
\end{tabular}

\subsection{Results of Three-point-bending Beam Test}

Typical F-CMOD curves in the four groups of specimens with different freeze-thaw cycles are shown in figure 2 , which can be divided into three stages. At the beginning of loading, the load $F$ of the beam basically increases linearly with the crack mouth opening displacement $C M O D$. In the groups with relatively low cycle (0 times, 25 times), the stage of the stable crack propagation is very short. When the maximum fracture load $F_{\max }$ is reached, $C M O D$ increases rapidly, while $F$ decreases rapidly. The fracture surface of the specimen is destroyed mainly due to the aggregate fracture as shown in figures 1 and 2. However, in the groups with high-cycle (50 times, 75 times), obvious stable crack propagation can be observed. When the load reaches the peak value, $F$ decreases rapidly. As shown in figure 1, on the fracture surface of the specimen, the failure is mainly the aggregate pullout. This is mainly because that in the process of freeze-thaw cycle, the bonding property between aggregate and slurry deteriorates, and the bonding capacity fails prematurely under low load. And then the failure mode is changed from aggregate fractured to pullout.

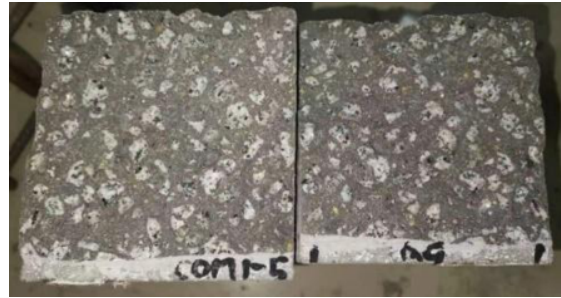

(a)

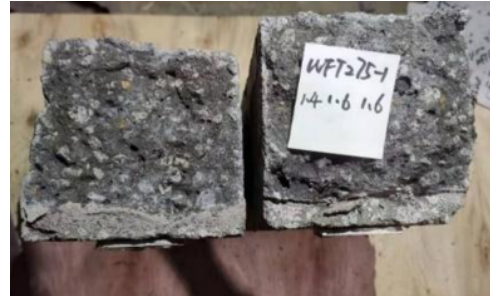

(b)

Figure 1. Typical fractured cross-section: (a) COM; (b) FT75.

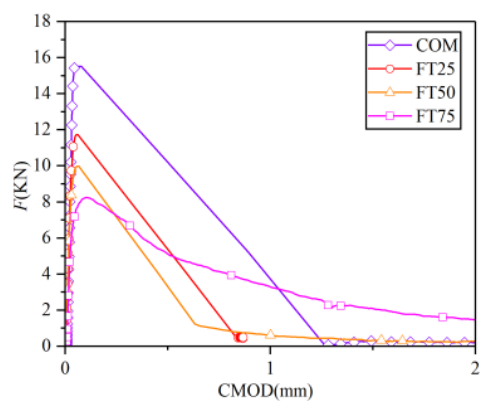

Figure 2. Typical P-CMOD curves. 


\section{Determination of $f_{\mathrm{t}}$ and $K_{\mathrm{IC}}$}

\subsection{Analytical Modelling}

For a three-point-bending notched beam, figure 3 shows the stress distribution on the cracked section in the mid-span under the maximum fracture load $F_{\max }$, where $a_{0}$ is the initial crack length, $b, h$ and $L$ are, respectively, the width, height and span length of the beam, and $L=2.5 \mathrm{~h}$. When the initial cracking state is reached, the crack becomes discontinuous due to the inhomogeneity of the material and the inevitable existence of micro cracks in the tip area. Also, the crack usually bypasses or passes through the aggregate. Therefore, the extent of crack propagation should be related to the maximum or average aggregate size of concrete. Precisely, the critical crack expansion length $\Delta a_{\mathrm{c}}$ should be integer or semi-integer in multiples of the maximum aggregate size $d_{\max }$. According to the study of $\mathrm{Hu}$ [12], the best estimate of $\Delta a_{\mathrm{c}}$ should be equal to the maximum aggregate particle size $d_{\max }$, i.e., $\Delta a_{\mathrm{c}}=d_{\max }$. Generelly, $d_{\max }=1.5 G$, where $G$ is the average aggregate size. It is assumed that the cohesive force is the nominal strength $\sigma_{\mathrm{n}}$ within a limited range of crack propagation length.

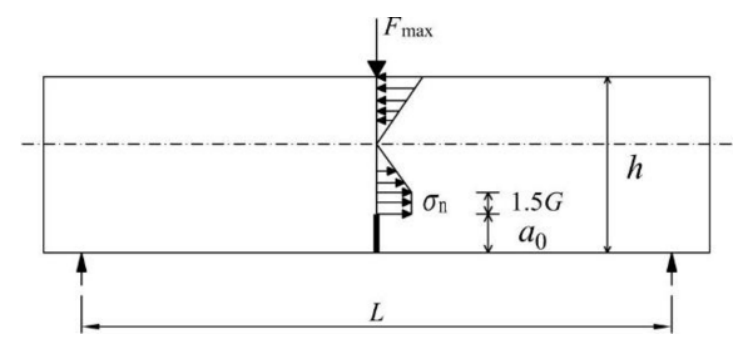

Figure 3. Stress distribution in the cracked section when the maximum load is reached.

Based on the assumption of linear strain distribution along the uncracked part in the critical section, the equilibrium condition of forces in Figure 3 gives the following equation according to the equilibrium condition of the forces in the section [13]:

$$
F_{\text {max }}+\frac{1}{2} W=\frac{2 b\left(h-a_{0}\right)\left(h-a_{0}+3 G\right)}{3 L} \sigma_{n}
$$

In (1), $F_{\max }$ is the maximum fracture load, and $W$ is the weight of the beam. The nominal strength $\sigma_{\mathrm{n}}$ can be expressed as follows:

$$
\begin{aligned}
& \sigma_{\mathrm{n}}=\frac{f_{\mathrm{t}}}{\sqrt{1+\frac{a_{\mathrm{e}}}{a_{\infty}^{*}}}} \\
& a_{e}=\left[\frac{(1-\alpha)^{2} Y(\alpha)}{1.12}\right]^{2} \sigma_{0}
\end{aligned}
$$




$$
\begin{aligned}
& \alpha=\frac{a_{0}}{h} \\
& Y(\alpha)=\frac{\left(1-2.5 \alpha+4.49 \alpha^{2}-3.98 \alpha^{3}+1.33 \alpha^{4}\right)}{(1-\alpha)^{3 / 2}} \\
& a_{\infty}^{*}=0.25\left(\frac{K_{I C}}{f_{t}}\right) \approx 3 G
\end{aligned}
$$

Substituting Eqs. (2) and (6) into Eq. (1), we can get the following:

$$
\begin{aligned}
& F_{\text {max }}+\frac{1}{2} W=f_{t} A \cdot g(h, \alpha, G) \\
& A=b h \\
& g(h, \alpha, G)=\frac{2 h(1-\alpha)\left(1-\alpha-\frac{3 G}{h}\right)}{3 L \sqrt{1+\frac{a_{e}}{3 G}}}
\end{aligned}
$$

Then

$$
\begin{aligned}
& f_{t}=\frac{F_{\max }+\frac{1}{2} W}{A \cdot g(h, \alpha, G)} \\
& K_{I C}=2 f_{t} \sqrt{3 G}
\end{aligned}
$$

Once the maximum fracture load $F_{\max }$ is given from the three-point bending test, the tensile strength $f_{\mathrm{t}}$ and fracture toughness $K_{\mathrm{IC}}$ without size effect can be determined via Eqs. (10) and (11), respectively.

\subsection{Results of $f_{t}$ and $K_{I C}$}

When the maximum fracture load $F_{\max }$ of each group of beams through the three-point bending test, $f_{\mathrm{t}}$ and $K_{\mathrm{IC}}$ can be determined through Eqs. (10) and (11), respectively. Since the maximum aggregate size of concrete is $10 \mathrm{~mm}$, so $G$ is adopted as $7 \mathrm{~mm}$. The values of $f_{\mathrm{t}}$ and $K_{\mathrm{IC}}$ obtained from $F_{\max }$ are shown in table 3. 
Table 3. Summary of $f_{\mathrm{t}}$ and $K_{\mathrm{IC}}$.

\begin{tabular}{|c|c|c|c|c|c|c|c|c|c|}
\hline $\begin{array}{l}\text { Nos. } \\
\text { of } \\
\text { group } \\
\text { S }\end{array}$ & $\alpha$ & $\begin{array}{l}F_{\max } \\
(\mathrm{kN})\end{array}$ & $\begin{array}{l}f_{\mathrm{t}} \\
(\mathrm{MPa})\end{array}$ & $\begin{array}{l}K_{\mathrm{IC}} \\
\left(\mathrm{MPa} \cdot \mathrm{m}^{1 /}\right. \\
\left.{ }^{2}\right)\end{array}$ & $\begin{array}{l}\text { Nos. } \\
\text { of } \\
\text { group } \\
\text { s }\end{array}$ & $\alpha$ & $\begin{array}{l}F_{\max } \\
(\mathrm{kN})\end{array}$ & $\begin{array}{l}f_{\mathrm{t}} \\
(\mathrm{MPa})\end{array}$ & $\begin{array}{l}K_{\mathrm{IC}} \\
\left(\mathrm{MPa} \cdot \mathrm{m}^{1}\right. \\
\left.{ }^{1}\right)\end{array}$ \\
\hline \multirow{22}{*}{ COM } & 0.09 & 15.917 & 6.24 & 1.808 & \multirow{22}{*}{ FT50 } & 0.09 & 11.529 & 4.58 & 1.328 \\
\hline & 0.10 & 16.429 & 6.70 & 1.943 & & 0.11 & 11.842 & 5.24 & 1.519 \\
\hline & 0.11 & 15.018 & 6.90 & 2.001 & & 0.11 & 12.118 & 4.88 & 1.414 \\
\hline & 0.11 & 14.637 & 6.60 & 1.914 & & 0.11 & 12.884 & 5.35 & 1.550 \\
\hline & 0.11 & 15.985 & 6.37 & 1.845 & & 0.12 & 11.410 & 4.97 & 1.440 \\
\hline & 0.12 & 14.526 & 6.06 & 1.757 & & 0.12 & 11.971 & 5.50 & 1.595 \\
\hline & 0.17 & 15.206 & 8.03 & 2.326 & & 0.14 & 11.385 & 5.10 & 1.478 \\
\hline & 0.20 & 14.417 & 7.38 & 2.139 & & 0.19 & 10.134 & 4.93 & 1.430 \\
\hline & 0.21 & 13.568 & 6.88 & 1.995 & & 0.19 & 9.543 & 4.60 & 1.332 \\
\hline & 0.21 & 12.738 & 6.38 & 1.848 & & 0.19 & 9.014 & 4.28 & 1.240 \\
\hline & 0.21 & 12.185 & 6.67 & 1.932 & & 0.20 & 9.474 & 4.68 & 1.356 \\
\hline & 0.22 & 12.099 & 6.18 & 1.792 & & 0.21 & 8.265 & 4.59 & 1.330 \\
\hline & 0.24 & 13.603 & 7.47 & 2.164 & & 0.22 & 9.410 & 5.55 & 1.608 \\
\hline & 0.25 & 10.526 & 5.93 & 1.719 & & 0.23 & 7.133 & 4.38 & 1.269 \\
\hline & 0.31 & 9.517 & 6.35 & 1.841 & & 0.26 & 7.985 & 4.65 & 1.347 \\
\hline & 0.31 & 10.637 & 7.56 & 2.191 & & 0.29 & 7.117 & 4.27 & 1.237 \\
\hline & 0.32 & 9.989 & 7.38 & 2.140 & & 0.29 & 7.538 & 4.83 & 1.400 \\
\hline & 0.33 & 8.989 & 6.95 & 2.013 & & 0.30 & 8.335 & 5.82 & 1.687 \\
\hline & 0.34 & 8.185 & 6.15 & 1.784 & & 0.32 & 7.892 & 5.63 & 1.630 \\
\hline & 0.35 & 10.738 & 7.44 & 2.158 & & 0.33 & 6.313 & 4.48 & 1.297 \\
\hline & \multirow[t]{2}{*}{0.37} & 9.517 & 7.54 & 2.185 & & 0.34 & 8.002 & 5.87 & 1.701 \\
\hline & & & $\begin{array}{l}6.82 \\
(0.60) \\
\end{array}$ & $\begin{array}{l}1.976 \\
(0.174)\end{array}$ & & & & $\begin{array}{l}4.96 \\
(0.50)\end{array}$ & $\begin{array}{l}1.438 \\
(0.145)\end{array}$ \\
\hline \multirow{22}{*}{ FT25 } & 0.07 & 14.313 & 6.07 & 1.758 & \multirow{22}{*}{ FT75 } & 0.07 & 11.510 & 4.29 & 1.244 \\
\hline & 0.07 & 16.737 & 6.11 & 1.770 & & 0.08 & 7.961 & 3.01 & 0.874 \\
\hline & 0.07 & 16.860 & 6.17 & 1.790 & & 0.08 & 9.146 & 3.99 & 1.156 \\
\hline & 0.08 & 12.47 & 5.09 & 1.474 & & 0.09 & 11.104 & 4.66 & 1.351 \\
\hline & 0.09 & 13.049 & 5.29 & 1.534 & & 0.09 & 9.077 & 3.65 & 1.057 \\
\hline & 0.10 & 13.438 & 6.13 & 1.776 & & 0.10 & 9.921 & 4.30 & 1.248 \\
\hline & 0.13 & 12.199 & 5.53 & 1.602 & & 0.12 & 9.939 & 4.19 & 1.214 \\
\hline & 0.14 & 9.943 & 4.33 & 1.255 & & 0.12 & 8.500 & 3.63 & 1.051 \\
\hline & 0.15 & 10.883 & 4.67 & 1.355 & & 0.12 & 9.555 & 4.43 & 1.285 \\
\hline & 0.15 & 11.588 & 5.12 & 1.483 & & 0.15 & 9.410 & 4.07 & 1.179 \\
\hline & 0.15 & 13.134 & 6.05 & 1.753 & & 0.15 & 8.406 & 3.80 & 1.101 \\
\hline & 0.17 & 10.884 & 5.01 & 1.453 & & 0.16 & 7.231 & 3.41 & 0.988 \\
\hline & 0.18 & 11.952 & 5.97 & 1.731 & & 0.16 & 7.568 & 3.33 & 0.966 \\
\hline & 0.22 & 11.015 & 6.40 & 1.854 & & 0.20 & 9.997 & 5.16 & 1.497 \\
\hline & 0.26 & 10.159 & 6.52 & 1.889 & & 0.22 & 6.237 & 3.40 & 0.986 \\
\hline & 0.26 & 8.992 & 5.00 & 1.449 & & 0.25 & 6.570 & 4.02 & 1.165 \\
\hline & 0.27 & 8.972 & 5.47 & 1.587 & & 0.28 & 7.927 & 4.98 & 1.444 \\
\hline & 0.31 & 9.848 & 6.33 & 1.834 & & 0.30 & 6.924 & 4.86 & 1.408 \\
\hline & 0.31 & 9.186 & 6.16 & 1.786 & & 0.30 & 7.524 & 4.93 & 1.430 \\
\hline & 0.32 & 8.776 & 5.73 & 1.661 & & 0.30 & 6.082 & 4.04 & 1.172 \\
\hline & \multirow[t]{2}{*}{0.33} & 8.533 & 6.37 & 1.846 & & 0.35 & 7.245 & 5.93 & 1.717 \\
\hline & & & $\begin{array}{l}5.69 \\
(0.63)\end{array}$ & $\begin{array}{l}1.649 \\
(0.183)\end{array}$ & & & & $\begin{array}{l}4.19 \\
(0.71)\end{array}$ & $\begin{array}{l}1.216 \\
(0.206)\end{array}$ \\
\hline
\end{tabular}




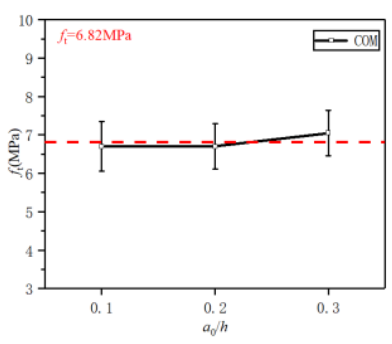

(a)

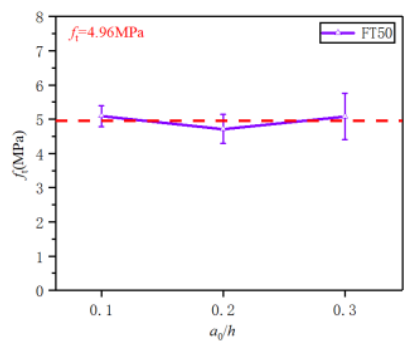

(c)

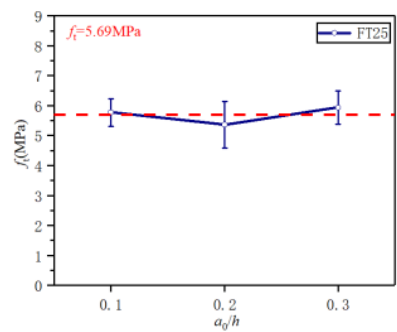

(b)

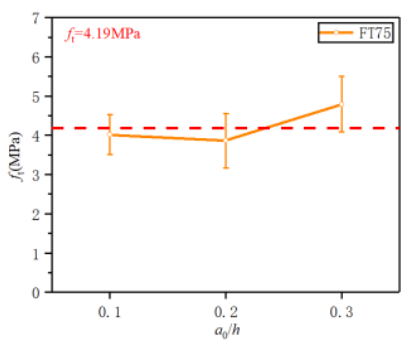

(d)

Figure 4. Variations $f_{\mathrm{t}}$ with of $a_{0} / h$ : (a) COM; (b) FT25; (c) FT50; (d) FT75.

Moreover, the variations of $f_{\mathrm{t}}$ with the $a_{0} / h$ are shown in Figure 4 . It should be noted that $f_{\mathrm{t}}$ obtained from the BEM can be regarded as the local tensile strength of the region of crack tip. Therefore, for each group of beams, the material properties at the crack tip will vary with $a_{0} / h$. Even for the same $a_{0} / h$, the measured $F_{\max }$ will be discrete, which would result in the variation in the calculated $f_{\mathrm{t}}$. But for each group of beams, the calculated value of $f_{\mathrm{t}}$ approaches one constant.

In Table 3, it can be seen that after 25, 50 and 75 freeze-thaw cycles, $f_{\mathrm{t}}$ is decreased by $16.6 \%, 27.3 \%$ and $38.6 \%$, and $K_{\mathrm{IC}}$ is decreased by $16.7 \%, 27.3 \%$ and $38.4 \%$ respectively. It can be found that the freeze-thaw cycle causes great damage to the concrete. Under the action of the osmotic pressure of pouring water in freeze-thaw cycles, the damage of concrete increases gradually, which leads to the decrease of $f_{\mathrm{t}}$ and $K_{\mathrm{IC}}$ with the increase of cycles.

\subsection{Discussion}

The splitting tensile test of concrete specimen was carried out for $28 \mathrm{~d}$ under the standard curing condition, where the obtained $f_{\mathrm{ts}}$ was $5.04 \mathrm{MPa}$ only, while $f_{\mathrm{t}}$ obtained using BEM [14] was $6.82 \mathrm{MPa}$. It is known that $f_{\mathrm{t}}$ is usually replaced by the splitting tensile strength $f_{\mathrm{t}}$, which is determined on the basis of linear elasticity under the assumption of continuity and homogeneity. In fact, the $f_{\text {ts }}$ only refers to the average tensile strength in the cross-section. However, concrete is a heterogeneous material, and the material inhomogeneity and discontinuity lead to uneven distribution of stress. However, $f_{\mathrm{t}}$ determined by BEM is an asymptotic value of the real tensile strength. So, 
it can be concluded that the real tensile strength of concrete must be greater than the average strength on the section.

Under the action of freeze-thaw cycles, the environment has a significant influence on $f_{\mathrm{t}}$ and $K_{\mathrm{IC}}$. After 75 times of freeze-thaw cycles, $f_{\mathrm{t}}$ and $K_{\mathrm{IC}}$ are decreased by more than $38 \%$. The main reason is that the solution freezes and expands in the surface of initial notch, which provides pressure on the crack wall as shown in Figure 5. As a result, the initial crack wall is repeatedly loaded and unloaded during the freeze-thaw cycle, leading to the deterioration of the concrete at the tip of the crack. In addition, the pressure on the wall gradually increases with increasing $a_{0} / h$, leading to severe deterioration of the specimen when $a_{0} / h=0.3$. It was found that a beam with $a_{0} / h$ higher than 0.3 would be damaged after the freeze-thaw cycle. Therefore, it is difficult to directly obtain the fracture parameters of specimens by using the quick freezing method with high $a_{0} / h$ after freeze-thaw cycles. With the introduction of BEM, the mean value and standard deviation of $f_{\mathrm{t}}$ can be calculated by using specimens with low $a_{0} / h$. Eq. (7) is used to predict the maximum fracture load $F_{\max }$ of specimens with high $a_{0} / h$.

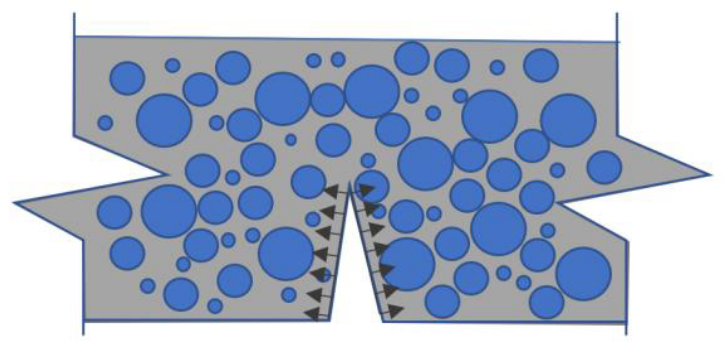

Figure 5. The pressure on the crack surface.

\section{Conclusions}

In this paper, fracture properties of small-size three-point-bending notched concrete beams with different initial notch lengths $(10 \mathrm{~mm}, 20 \mathrm{~mm}, 30 \mathrm{~mm})$ are analyzed under different freeze-thaw cycles $(0,25,50,75)$. Furthermore, considering the discontinuity and inhomogeneity of materials, the critical effective crack length under $F_{\max }$ at the crack tip is expressed as the product of the average aggregate size of concrete and the discrete coefficient reflecting the discontinuity of crack propagation. By using the maximum fracture load from the test, $f_{\mathrm{t}}$ and $K_{\mathrm{IC}}$ were determined, and the influence of freeze-thaw cycle on the concrete performance was analyzed. The specific conclusions are as follows:

(1) For concrete specimens under the same condition, there is certain difference in $f_{\mathrm{t}}$ and $K_{\mathrm{IC}}$ obtained against different $\alpha$, but each tends to be constant.

(2) With the increase in the cycles, $f_{\mathrm{t}}$ and $K_{\mathrm{IC}}$ showed decreasing trends. After 25 , 50 and 75 freeze-thaw cycles, $f_{\mathrm{t}}$ was decreased by $16.6 \%, 27.3 \%$ and $38.6 \%$, respectively, while $K_{\mathrm{IC}}$ was decreased by $16.7 \%, 27.3 \%$ and $38.4 \%$, respectively, indicating that the erosion of the freeze-thaw cycle has a significant effect on the deterioration of the concrete crack resistance. 
(3) The tensile strength obtained based on BEM is the true strength without size effect, and its value is significantly higher than the splitting tensile strength $f_{\mathrm{ts}}$ which represents the traditional tensile strength of concrete.

(4) With the increase in the freeze-thaw cycles, the internal defects of concrete increase, and its discontinuity and inhomogeneity becomes more significant. In this paper, $f_{\mathrm{t}}$ and $K_{\mathrm{IC}}$ are determined based on the BEM by fully considering the influence of material discontinuity and inhomogeneity. Therefore, the degrees of deterioration and damage of concrete after the freeze-thaw action can be estimated more rationally.

\section{References}

[1] Gu ZQ. Experimental research on strength and fracture properties of hydraulic concrete after threefactor deterioration. Zhengzhou University; 2015. (In Chinese)

[2] Hu SW and Wang Y. Experimental study on double-K fracture toughness of concrete in different freezing and thawing modes. Hydro-Science and Engineering. 2018; (2): 90-96 (In Chinese)

[3] Wang JR, Chen YL and Fu Y. Study on fracture properties of different pre-crack concreteunder freezing and thawing environment. Journal of Water Resources and Water Engineering. 2019; 30(2): $178-185$.

[4] Zhao YR, Song B, Wang L and Han XF. Fracture properties of basalt fiber reinforced concrete after freeze-thaw cycles. Journal of Building Materials. 2019; 22(4): 575-583. (In Chinese)

[5] Xu SL and Reinhardt HW. Crack extension resistance and fracture properties of quasi-brittle softening materials like concrete based on the complete process of fracture. International Journal of Fracture. 1998; 92(1): 71-99.

[6] Xu SL and Reinhardt HW. Determination of double- K criterion for crack propagation in quasi-brittle fracture, Part II: Analytical evaluating and practical measuring methods for three-point bending notched beams .International Journal of Fracture. 1999; 98(2): 151-177.

[7] Bažant ZP and Kazemi MT. Determination of fracture energy, process zone longth and brittleness number from size effect, with application to rock and conerete. International Journal of Fracture. 1990; 44(2): 111-131.

[8] Bažant ZP. Size effect in blunt fracture: concrete, rock, metal. Journal of Engineering Mechanics. 1984; 110(4): 518-535.

[9] $\mathrm{Hu} \mathrm{XZ}$ and Duan K. Influence of fracture process zone height on fracture energy of concrete. Cement and Concrete Research. 2004; 34(8): 1321-1330.

[10] $\mathrm{Hu} \mathrm{XZ}$ and Duan K. Size effect and quasi-brittle fracture: the role of FPZ. International Journal of Fracture. 2008; 154(1-2): 3-14.

[11] Wang BH, Hu XZ, Hui JZ, Lu PM and Jiang B. CNT-reinforced adhesive joint between grit-blasted steel substrates fabricated by simple resin pre-coating method. The Journal of Adhesion. 2018; 94(7): 529-540.

[12] Han X, Chen Y, Hu X, Liu W, Li Q and Chen S. Granite strength and toughness from small notched three-point-bend specimens of geometry dissimilarity. Engineering Fracture Mechanics. 2019; 216; 106482.

[13] Yang ST, Zhang XS, Yu M and Yao J. An analytical approach to predict fracture parameters of coral aggregate concrete immersed in seawater. Ocean Engineering. 2019; 191; 106508.

[14] $\mathrm{Hu} \mathrm{XZ} \mathrm{and} \mathrm{Folker} \mathrm{W.} \mathrm{Size} \mathrm{effect} \mathrm{on} \mathrm{toughness} \mathrm{induced} \mathrm{by} \mathrm{crack} \mathrm{close} \mathrm{to} \mathrm{free} \mathrm{surface.} \mathrm{Engineering}$ Fracture Mechanics. 2000; 65(2): 209-221. 